\title{
LIST OF PARTICIPANTS
}

ADAMS, N.G., Space Res. Dept., Univ. of Birmingham, Birmingham, England.

ALlAMANDOLA, L.J., Huygens Lab., Wassenaarseweg 78, Leiden 2300 RA, The Netherlands.

ANDREW, B.H., Herzberg Inst. of Astrophysics, NRC, Ottawa KlA OR6, Canada.

ANICICH, V.G., Jet Propulsion Lab., 4800 Oak Grove Dr., Pasadena, CA 91103, U.S.A.

ARNOLD, F., Max-Planck-Inst. für Kernphysik, 69 Heidelberg, F.R. Germany.

ASUNDI, R.K., Spectroscopy Divn., BARC, Trombay, Bombay-85, India 400085.

AVERY, L.W., Herzberg Inst. of Astrophysics, NRC, Ottawa KlA OR6, Canada.

BALLY, J., Dept. of Astronomy, Univ. of Massachusetts, Amherst, MA 01003 , U.S.A.

BAR-NUN, A., Dept. of Geophysics \& Planetary Sc., Tel Aviv Univ., Tel Aviv, Israel.

BARRETT, A.H., Massachusetts Inst. Tech., Cambridge, MA 02139, U.S.A.

BARRETT, J., Physics Dept. State Univ. of New York, Stony Brook, NY 11794 , U.S.A.

BAUDRY, A., Obs. de Bordeaux, 33270 Floirac, France.

BECKWITH, S., Dept. of Astronomy, Space Sc. Bldg., Cornell Univ., Ithaca, NY 14853, U.S.A.

BEICHMAN, C.A., California Inst. Tech., Pasadena, CA 91125, U.S.A. BETZ, A.L., Dept. of Physics, Univ. of California, Berkeley, CA 94720, U.S.A.

BIEGING, J.H., Radio Astronomy Lab., Univ. of California, Berkeley, CA 94720, U.S.A.

BIERMANN, L., Max-Planck-Inst. für Physik, Föhringer Ring 6, 8000 München 40, F.R. Germany.

BLACK, J.H., Center for Astrophysics, 60 Garden St., Cambridge, MA 02138, U.S.A.

BLITZ, L., Radio Astronomy Lab., Univ. of California, Berkeley, CA 94720 , U.S.A.

BOHME, D.K., Dept. of Chemistry, York Univ., Downsview, Ontario, Canada.

BOK, B.J., Steward Obs., Univ. of Arizona, Tucson, AZ 85721, U.S.A.

B0OTH, R.S., Nuffield Radio Astronomy Lab., Jodrell Bank, Macclesfield, England.

BROTEN, N.W., Herzberg Inst. of Astrophysics, NRC, Ottawa KlA 0R6, Canada.

BUHL, D., Code 693, NASA Goddard Space Flight Center, Greenbelt, MA 20771. U.S.A.

BURDYUZHA, V.V., Space Res. Inst., USSR Academy of Sc., Prof souznaya 84, Moscow, USSR.

BUXTON, R., Massachusetts Inst. Tech. Cambridge, MA 02139, U.S.A.

CARLSON, W.J., Dept. of Astronomy, Ohio State Univ., Columbus, $\mathrm{OH} 43210$, U.S.A. 
CARRUTHERS, G.R., Code 7123, Naval Res. Lab., Washington, DC 20375, U.S.A.

CHAFFEE, F.H., Mt. Hopkins Obs., P.0. Box 97, Amado, AZ 85640, U.S.A. CHIN, G., Code 693, NASA Goddard Space Flight Center, Greenbelt, MD 20771, U.S.A.

CHURCHWELL, E.B., Dept of Astronomy, Univ. of Wisconsin, Madison, WI 53706, U.S.A.

CLARK, F.0., Univ. of Kentucky, Dept. Physics \& Astronomy, Lexington, KY 40506, U.S.A.

COHEN, R., Goddard Inst. for Space Studies, 2800 Broadway, New York, NY 10025, U.S.A.

COLOMB, F.R., Inst. Argentino de Radioastronomia, Casilla 5, 1894 Villa Elisa, Buenos Aires, Argentina.

CONG, H-I., Goddard Inst. for Space Studies, 2880 Broadway, New York, NY 10025, U.S.A.

CROVISIER, J., Dépt. de Radioastronomie, Obs. de Meudon, 92190 Meudon, France.

CRUTCHER, R.M., Astronomy Dept., Univ. of Illinois, Urbana, IL 61801 , U.S.A.

CUDABACK, D.D., Radio Astronomy Lab., Univ. of California, Berkeley, CA 94710, U.S.A.

CUMMINS, S.E., Goddard Inst. for Space Studies, 2880 Broadway, New York, NY 10025, U.S.A.

DALGARNO, A., Harvard College Obs., 60 Garden St., Cambridge, MA 02138, U.S.A.

DAME, T.M., Pupin Hall, Columbia University, New York, NY 10027 , U.S.A.

DANKS, A.C., European Southern Obs., Casilla 16317, Santiago 9, Chile.

DAVIES, R.D., Nuffield Radio Astronomy Lab., Jodrell Bank, Macclesfield, England.

DE JONG, T., Astronomical Inst., Roeterstraat 15, Amsterdam, The Netherlands.

DE ZAFRA, R., Physics Dept. State Univ. of New York, Stony Brook, NY 11794, U.S.A.

DICKEL, H.R., Astronomy Dept., Univ. of Illinois, Urbana, IL 61801, U.S.A.

DICKINSON, D.F., Jet Propulsion Lab., 4800 Oak Grove Dr., Pasadena, CA 91103, U.S.A.

DIERCKSEN, G.H.F., Max-Planck-Inst. für Physik und Astrophysik, Föhringer Ring 6, D8000 München 40, F.R. Germany.

DINGER, A.S., Jet Propulsion Lab., 4800 Oak Grove Dr., Padadena, CA 91103, U.S.A.

DOHERTY, L.H., Herzberg Inst. of Astrophysics, NRC, Ottawa K1A 0R6, Canada.

DOWNES, D., Max-Planck-Inst. für Radioastronomie, Auf dem Hügel 69 , D5300 Bonn 1, West F.R. Germany.

DRAPATZ, S., Max-Planck-Inst. für Extraterrestrische Physik, 8046 Garching, F.R. Germany.

DULEY, W.W., Dept. of Physics, York University, Downsview, Ontario M3J 1P3, Canada. 
DYMANUS, A., Grameystr 35, Ni jmegen, The Netherlands.

ELITZUR, M., Dept. of Physics, Univ. of Illinois, Urbana, IL 61801, U .S.A.

ELMEGREEN, B.G., Dept. of Astronomy, Columbia Univ., New York, NY 10027, U.S.A.

EVANS, N.J., Dept. of Astronomy, Univ. of Texas, Austin, TX 78721, U.S.A.

EVENSON, K.M., National Bureau of Standards, Boulder, CO 80302 , U.S.A.

FALGARONE, E., Dept. of Radioastronomie, Obs. de Paris, F-92190 Meudon, France.

FEDERMAN, S., Dept of Astronomy, Univ. of Texas, Austin, TX 78712, U.S.A.

FEHSENFELD, F.C., Aeronomy Lab., NOAA Environmental Res. Lab., Boulder, CO 80302, U.S.A.

FELDMAN, P.A., Herzberg Inst. of Astrophysics, NRC, Ottawa KlA OR6, Canada.

FIELD, D., School of Chemistry, Cantocks Close, Univ. of Bristol, Bristol, England.

FITTON, B., Astronomy Divn., European Space Agency, 2200 AA Noordwijk, The Netherlands.

FORSTER, J.R., Radio Sterrenwacht Dwingeloo, 7990 AA Dwingeloo, The Netherlands.

Fox, K., Dept. of Physics \& Astronomy, Univ. of Tennessee, Knoxville, TN 37916, U.S.A.

FRISCH, P.C., Univ. of Chicago, Enrico Fermi Inst., 933 E 56 th St., Chicago, IL 60637, U.S.A.

FUKUI, Y., Tokyo Astronomical Obs., Mitaka, Tokyo, Japan.

GEBALLE, T.R., Hale Obs., 813 Santa Barbara St., Pasadena, CA 91101, U.S.A.

GEZARI, D.Y., NASA Goddard Space Flight Center, Code 693.2, Greenbelt, MD 20771 , U.S.A.

GILLESPIE, A.R., Max-Planck-Inst. für Radioastronomie, Auf dem Hügel 69, D5300 Bonn 1, F.R. Germany.

GILMORE, W., Dept. of Astronomy, Univ. of Toronto, Toronto, Canada.

GILRA, D.P., Kapteyn Astronomical Inst., Univ. of Groningen, Groningen, The Netherlands.

GLASSGOLD, A.E., Physics Dept., New York Univ., 4 Washington P1., New York, NY 10063, U.S.A.

GOLD, T., Center for Radiophysics and Space Res., Cornell Univ., Ithaca, N.Y. 14853, U.S.A.

GOLDREICH, P., Divn. of Geological \& Planetary Sc., California Inst. Tech., Pasadena, CA 91125, U.S.A.

GOLDSMITH, P.F., Dept. of Astronomy, Univ. of Massachusetts, Amherst, MA 01003, U.S.A.

GOMEZ-GONZALEZ, J., Obs. Astronomico Nacional, Al fonso XII N3, Madrid, - Spain.

GORDON, M.A., National Radio Astronomy Obs., 2010 North Forbes Blvd., Tucson, AZ 85705, U.S.A.

GoSS, W.M., Kapteyn Astronomical Inst., Univ. of Groningen, Groningen, The Netherlands. 
GOTTLIEB, C.A., Goddard Inst. for Space Studies, 2880 Broadway, New York, NY 10025, U.S.A.

GREENBERG, J.M., Huygens Lab., Wassenaarseweg 78, Leiden 2300 RA, The Netherlands.

GUELIN, M., Obs. de Paris, Section d'Astrophysique, 92190 Meudon, France.

HAGEN, W., Huygens Lab., Wassenaarseweg 78, Leiden, 2300 RA, The Netherlands.

HALL, D.N.B., Kitt Peak Natinal Obs., 950 North Cherry Av., Tucson, AZ 85726, U.S.A.

HARTLEY, C.H., Electronic Space Systems Corp., Old Powder Mill Rd., Concord, MA 01742, U.S.A.

HERBST, E., Dept. of Chemistry, College of William \& Mary, Williamsburg, VA, U.S.A.

HERZBERG, G., Herzberg Inst. of Astrophysics, NRC, Ottawa KlA OR6, Canada.

HIGGS, L.A., Herzberg Inst. of Astrophysics, NRC, Ottawa K1A 0R6, Canada.

HILLS, R.E., Cavendish Lab., Cambridge Univ., Madingley Rd., Cambridge, England.

HJALMARSON, A.G., Onsala Space Obs., S-43034 Onsala, Sweden. HO, P.T.P., Dept. of Astronomy Univ. of Massachusetts, Amherst, MA 01003 , U.S.A.

HOGLUND, B., Onsala Space Obs., S-43034 Onsala, Sweden.

HOLLENBACH, D.J., NASA Ames Research Center, Moffett Field, CA 94035, U.S.A.

HOLLIS, J.M., National Radio Astronomy Obs., 2010 North Forbes Blvd., Tucson, AZ 85705, U.S.A.

HUEBNER, W.F., Los Alamos Scientific Lab., Los Alamos, NM 87545, U.S.A.

HUGUENIN, R., Dept of Astronomy, Univ. of Massachusetts, Amherst, MA 01003, U.S.A.

HUNTRESS, W.T., Jet Propulsion Lab., 4800 Oak Grove Dr., Pasadena, CA 91103, U.S.A.

IRVINE, W.M., Onsala Space Obs., S-43034 Onsala, Sweden.

JEFFERS, S., Physics Dept., York Univ., Downsview, Ontario M3J 1P3, Canada.

JENNINGS, D.E., Code 693, NASA Goddard Space Flight Center, Greenbelt, MD 10771, U.S.A.

JOHNS, J.W.C., Herzberg Inst. of Astrophysics, NRC, Ottawa KlA 0R6, Canada.

JOHNSON, D.R., National Bureau of Standards, Washington, DC 20234 , U.S.A.

JOHNSTON, K.J., Code 7134, Naval Res. Lab., Washington, DC 20375, U.S.A.

KEGEL, W.H., Inst. für Theoretische Physik, Univ. of Frankfurt, Frankfurt, F.R. Germany.

KESTEVEN, M.J., Dept. of Physics, Queen's University, Kingston, Canada.

KIRBY, K. , Harvard-Smithsonian Center for Astrophysics, 60 Garden St., Cambridge, MA 02138, U.S.A. 
KOLLBERG, E., Onsala Space Obs,, S-43034 Onsala, Sweden.

KRAEMER, W.P., Max-Planck-Inst. für Physik und Astrophysik, Föhringer Ring 6, 8 München, F.R. Germany.

KRASSNER, J., Grumman Aerospace Corp. Plant 26, Bethpage, NY 11714 , U.S.A.

KROTO, H.W., Dept. of Chemistry, Univ. of Sussex, Brighton, BNl 9QT, England.

KUIPER, T.B.H., Jet Propulsion Lab., California Inst. of Tech., Pasadena, CA 91103, U.S.A.

KUTNER, M.L., Physics Dept., Rensselaer Polytechnic Inst., Troy, NY 12181, U.S.A.

KWOK, S., Herzberg Inst. of Astrophysics, NRC, Ottawa KlA OR6, Canada.

LANE, A.P., Dept. of Ast ronomy, Univ. of Massachusetts, Amherst, MA 01003, U.S.A.

LANGER, W.D., Dept. of Physics \& Astronomy, Univ. of Massachusetts, Amherst, MA 01003, U.S.A.

LEES, R.M., Dept. of Physics, Univ. of New Brunswick, Fredericton, E 3B 5A3, Canada.

LEPINE, J.R.D., CRAAM, Obs. Nacional, Rua Para 277, Sao Paulo-01243, Brazil.

LEUNG, C.M., Dept. of Physics, Renssalaer Polytechnic Inst., Troy, NY 12181 , U.S.A.

LINKE, R.A., Bell Labs., Crawford Hill Lab., Holmdel, NJ 07733, U.S.A.

LISZT, H.S., National Radio Astronomy Obs., Edgemont Rd., Charlot tesville, VA 22901 , U.S.A.

LITVAK, M.M., Jet Propulsion Lab., 4800 Oak Grove Dr., Pasadena, CA 91103, U.S.A.

LO, K.Y., California Inst. Tech., Pasadena, CA 91125, U.S.A.

LOCKE, J.L., Herzberg Inst. of Astrophysics, NRC, Ottawa K1A OR6, Canada.

LORTET, M.C., D.A.F., Obs. de Meudon, 92190 Meudon, France.

LOVAS, F.J., Molecular Spectroscopy Divn., National Bureau of Standards, Washington, DC 20234, U.S.A.

LUCAS, R., Radioastronomie, Ecole Normale Superieure, 75230 Paris Cedex 05, France.

LUTZ, B.L., Lowell Obs., P.0. Box 1269, Flagstaff, AZ 86002, U.S.A.

MACDONALD, G.H., Electronics Lab., Univ. of Kent, Canterbury, Kent CT2 7NT, England.

MACKAY, G., Dept. of Chemistry, York Univ., Downsview, Canada.

MacLEOD, J.M., Her zberg Inst. of Astrophysics, NRC, Ot tawa K1A OR6, Canada.

MARQUES DOS SANTOS, P., Obs. Nacional, CRAAM, Rua Para 277, Sao Paulo 01243 Brazil.

MATHESON, D.N., SRC Appleton Lab., Chilbolton Obs., Stockbridge,

- Hants, England.

McCABE, E.M., Astronomy Centre, Univ. of Sussex, Brighton, BN1 9QJ, England.

MCCARROLL, R., Lab. d'Astrophysique, Univ. de Bordeaux, 33405-Talence, France. 
McCUTCHEON, W.H., Dept. of Physics, Univ. of British Columbia, Vancouver, V6T 1W5, Canada.

McEWAN, M.J., Jet Propulsion Lab., 4800 Oak Grove Dr., Pasadena, CA 91103, U.S.A.

McLAREN, R.A., Dept. of Astronomy, Univ, of Toronto, Toronto, M5S 1A7, Canada.

MEZGER, P.G., Max-Planck-Inst. für Radioastronomie, Auf dem Hugel 69, 53 Bonn 1, F.R. Germany.

MILLAR, T.J., Dept. of Theoretical Physics, Univ. of Oxford, Oxford OXl 3NP, England.

MINN, Y.K., National Astronomical Obs., Yoksam-dong, Kangnam-Ku, Seoul, Korea.

MITCHELL, G.F., Jet Propulsion Lab., 4800 Oak Grove Dr., Pasadena, CA 91103, U.S.A.

MORAN, J.M., Center for Astrophysics, 60 Garden St., Cambridge, MA 02138, U.S.A.

MORIMOTO, M., Tokyo Astronomical Obs., Mitaka, Tokyo, Japan.

MORRIS, M., Astronomy Dept., Columbia Univ., New York, NY 10027 , U.S.A.

MOUSCHOVIAS, T.Ch., Dept. of Physics \& Astronomy, Univ. of Illinois, Urbana, IL 61801, U.S.A.

MYERS, P.C., Massachusetts Inst. Tech., Cambridge, MA 02139, U.S.A.

NADEAU, D., California Inst. Tech., Pasadena, CA 91125, U.S.A.

NAKAGAWA, N., Dept. of Chemistry, Univ. of Electro-communicatins, Chofu, Tokyo, Japan.

NORMAN, C.A., Huygens Lab., Wassenaarseweg 78, Leiden 2300 RA, The Netherlands.

OKA, T., Herzberg Inst. of Astrophysics, NRC, Ottawa KlA OR6, Canada. OMONT, A., CERMO, B.P. 53, 38041 Grenoble-Cedex, France.

PALMER, P., Dept. of Astronomy, Univ. of Chicago, 1100 E 58 th St., Chicago, IL 60637, U.S.A.

PANKONIN, V., Divn. of Astronomical Sc., NSF, 1800 G St. N.W., Washington, DC 20550, U.S.A.

PARRISH, A., FCRAO, Univ. of Massachusetts, Amherst, MA 01003, U.S.A. PAULS, T., Max-Planck-Inst. für Radioastronomie, Auf dem Hügel 69, 5300 Bonn 1, F.R. Germany.

PENZIAS, A.A., Bell Labs., Crawford Hill Lab., Box 400, Holmdel, NJ 07733, U.S.A.

PHILLIPS, T.G., Bell Labs., Murray Hi11, NJ 07974, U.S.A.

PLAMBECK, R.L., Radio Astronomy Lab., Univ. of California, Berkeley, CA 94720 , U.S.A.

POPPEL, W., Inst. Argentino de Radioastronomia, Casilla 5, 1894 Villa Elisa, Buenos Aires, Argentina.

PRASAD, S.S., Jet Propulsion Lab., California Inst. Tech., Pasadena, CA 91103, U.S.A.

RADFORD, H.E., Harvard College Obs., 60 Garden St., Cambridge, MA 02138, U.S.A.

RADHAKRISHNAN, V., Raman Research Inst., Bangalore 56006, India. RAMSAY, D.A., Herzberg Inst. of Astrophysics, NRC, Ot tawa KlA OR6, Canada. 
RICKARD, L.J., National Radio Astronomy Obs., Edgemont Road, Charlot tesville, VA 22901, U.S.A.

RIDGWAY, S.T., Kitt Peak National Obs., Tucson, AZ 85726, U.S.A. RIEU, N.Q., Obs. de Meudon, 92190 Meudon, France.

ROBINSON, B.J., CSIRO Radiophysics, P.O. Box 76, Epping 2121, Australia.

RODRIGUEZ-KUIPER, E.N., Ba11 Aerospace Systems Divn., Western Labs., Gardena, California, U.S.A.

ROUEFF, E., DAPHE, Obs. de Meudon, 92190 Meudon, France.

RUBIN, R.H., Physics Dept., California State Univ. at Fullerton, Fullerton, CA 92634, U.S.A.

RYDBECK, G., Onsala Space Obs., S-43034 Onsala, Sweden.

SAKATA, A., The Univ. of Electro-communications, Chofu, Tokyo, Japan. SANDERS, D.B., Dept. of Earth \& Space Sc., State Univ. of New York, Stony Brook, NY 11733, U.S.A.

SANDQVIST, Aa., Stockholm Obs., S-13300, Saltsjöbaden, Sweden.

SCALISE, E., Obs. Nacional, CRAAM, Rua Para 277, 01243 Sao Paulo, Brazil.

SCHIFF, H.I., Dept of Chemistry, York Univ., Downsview, Canada.

SCHLOERB, F.P., Astronomy Program, Univ. of Massachusetts, Amherst, MA 01003 , U.S.A.

SCHWARTZ, P.R., Naval Res. Lab., Code 7138, Washington, DC 20375, U.S.A.

SCOTT, P.F., Cavendish Lab., Cambridge Univ., Madingley Road, Cambridge, England.

SCOVILlE, N.Z, Dept. of Physics \& Astronomy, Univ. of Massachusetts, Amherst, MA 01002, U.S.A.

SHERWOOD, W.A., Max-Planck Inst. für Radioastronomie, Auf den Hugel 69, 53 Bonn, F.R. Germany.

SHIVANANDAN, K., Code 7122.1 Space Sc. Divn., Naval Res. Lab., Washing ton, DC, U.S.A.

SHUTER, W.L.H., Dept. of Physics, Univ. of British Columbia, Vancouver V6T lW5, Canada.

SILK, J.I., Dept. of Astronomy, Univ. of California, Berkeley, CA 94720, U.S.A.

SLYSH, V.I., Space Res. Inst., Profsoyuznaya 84, Moscow, U.S.S.R.

SMITH, D., Space Res. Dept., Univ. of Birmingham, Birmingham B15 2TT, England.

SMITH, P.L., Harvard-Smithsonian Center for Astrophysics, 60 Garden St., Cambridge, MA 01138, U.S.A.

SNELL, R.L., Astronomy Dept., Univ. of Massachusetts, Amherst, MA 01003, U.S.A.

SNOW, T.P., LASP, Univ. of Colorado, Boulder CO 80309, U.S.A.

SNYDER, L.E., Astronomy Dept., Univ. of Illinois, Urbana, IL 61801 , U.S.A.

SOLLNER, T.C.L.G., FCRAO, Univ. of Massachusetts, Amherst, MA 01003 , U.S.A.

SOLOMON, P.M., Astronomy Program, State Univ. of New York, -Stony Brook, NY 11794, U.S.A.

SOMERVILLE, W.B., Dept. Physics \& Astronomy, Univ. College, London, England. 
STAUDE, H.J., Max-Planck Inst. für Astronomie, 69 Heidelberg Königstuhl, F.R. Germany.

STOREY, J.W.V., Physics Dept., Univ. of California, Berkeley, CA 94720 , U.S.A.

SUZUKI, H., Dept. of Physics, Kyoto Univ., Kyoto, Japan.

TATUM, J.B., Dept. of Physics, Univ. of Victoria, Victoria, V8W 2Y2, Canada.

TENOR IO-TAGLE, G., ESO/CERN, Ch. 1211 Geneve 23, Switzerland. THADDEUS, P., Goddard Inst. for Space Studies, 2800 Broadway, New York, NY 10025, U.S.A.

TIELENS, A.G.G.M., Sterrewacht, Wassenaarseweg 78, Leiden 2300 RA, The Netherlands.

TOWNES, C.H., Dept. of Physics, Univ. of California, Berkeley, CA 94720, U.S.A.

VANDEN BOUT, P., Astronomy Dept., Univ. of Texas, Austin, TX 78712, U.S.A.

VANYSEK, V., Dept. of Astronomy \& Astrophysics, Charles Univ., Svedska 8, 15000 Prague 5, Smichov, Czechoslovakia.

WALMSLEY, M.C., Max-Planck Inst. für Radioastronomie, Auf dem Hugel 69, 53 Bonn, F.R. Germany.

WANNIER, P.G., California Inst. Tech., Pasadena, CA 91125, U.S.A.

WATANABE, T., Space Environment Lab., NOAA Environmental Res. Lab., Boulder, CO, U.S.A.

WATSON, J.K.G., Herzberg Inst. of Astrophysics, NRC, Ottawa KlA OR6, Canada.

WATSON, W.D., Depts . of Physics \& Astronomy, Univ. of Illinois, Urbana, IL 61801 , U.S.A.

WELCH, W.J., Radio Astronomy Lab., Univ. of California, Berkeley, CA 94720 , U.S.A.

WHITE, R., Five College Astronomy Dept., Smith College, Northampton, MA 01063, U.S.A.

WILLIS, C., Chemistry Divn., National Research Council, Ottawa K1A OR6, Canada.

WILLNER, S.P., Physics Dept., Univ. of California, San Diego, La Jolla, CA 92093, U.S.A.

WILSON, R.W., Bell Labs., Holmdel, NJ 07733, U.S.A.

WILSON, T.L., Max-Planck Inst. für Radioastronomie, Auf Dem Hügel 69, D53 Bonn 1, F. R. Germany.

WINNEWISSER, G., Max-Planck Inst. für Radioastronomie, Auf Dem Hügel 69, D53 Bonn 1, F.R. Germany.

WOODS, R.C., Dept. of Chemistry, Univ. of Wisconsin, Madison, WI 53706, U.S.A.

WOOTTEN, H.A., Owens Valley Radio Obs., California Inst. Tech., Pasadena, CA 91125, U.S.A.

WRIGHT, J.P., National Sc. Foundation, 1800 E. St. N.W., Washing ton, DC 20550, U.S.A.

WYNN-WILLIAMS, C.G., Inst. for Astronomy, Univ. of Hawaii, 2680 Woodlawn Dr., Honolulu, HI 96822, U.S.A.

ZUCKERMAN, B., Dept. of Physics \& Astronomy, Univ. of Maryland, College Park, MD, U.S.A. 